\title{
Comparison of CT number calibration techniques for CBCT- based dose calculation
}

\author{
Alex Dunlop - Dualta McQuaid - Simeon Nill · Julia Murray · \\ Gavin Poludniowski • Vibeke N. Hansen · Shreerang Bhide • \\ Christopher Nutting · Kevin Harrington · Kate Newbold · Uwe Oelfke
}

Received: 19 May 2015 / Accepted: 19 August 2015 / Published online: 24 September 2015

(C) The Author(s) 2015. This article is published with open access at Springerlink.com

\begin{abstract}
Purpose The aim of this work was to compare and validate various computed tomography (CT) number calibration techniques with respect to cone beam CT (CBCT) dose calculation accuracy.

Methods CBCT dose calculation accuracy was assessed for pelvic, lung, and head and neck $(\mathrm{H} \& \mathrm{~N})$ treatment sites for two approaches: (1) physics-based scatter correction methods $\left(\mathrm{CBCT}_{\mathrm{r}}\right)$; (2) density override approaches including assigning water density to the entire CBCT (W), assignment of either water or bone density (WB), and assignment of either water or lung density (WL). Methods for CBCT density assignment within a commercially available treatment planning system $\left(\mathrm{RS}_{\text {auto }}\right)$, where $\mathrm{CBCT}$ voxels are binned into six density levels, were assessed and validated. Dose-difference maps and dose-volume statistics were used to compare the
\end{abstract}

Dr. A. Dunlop, PhD $(\bowtie) \cdot J$. Murray, FRCR · S. Bhide, FRCR · K. Harrington, FRCR $\cdot$ K. Newbold, FRCR

The Royal Marsden Hospital,

Sutton, Surrey SM2 5PT, Downs Road, UK

e-mail: alex.dunlop@rmh.nhs.uk

S. Nill, PhD · D. McQuaid, PhD · Dr. A. Dunlop, PhD · V.

N. Hansen, $\mathrm{PhD} \cdot \mathrm{U}$. Oelfke, PhD

Joint Department of Physics, Institute of Cancer Research, The

Royal Marsden NHS Foundation Trust,

London SM2 5NG, UK

J. Murray, FRCR · S. Bhide, FRCR · C. Nutting, FRCR ·

K. Harrington, FRCR

The Institute of Cancer Research,

London SM2 5NG, UK

G. Poludniowski, $\mathrm{PhD}$

Department of Medical Physics, Karolinska University Hospital,

Stockholm 171 76, Sweden
CBCT dose distributions with the ground truth of a planning $\mathrm{CT}$ acquired the same day as the CBCT.

Results For pelvic cases, all CTN calibration methods resulted in average dose-volume deviations below $1.5 \%$. RSauto provided larger than average errors for pelvic treatments for patients with large amounts of adipose tissue. For H\&N cases, all CTN calibration methods resulted in average dose-volume differences below $1.0 \%$ with $\mathrm{CBCT}_{\mathrm{r}}(0.5 \%)$ and $\mathrm{RS}_{\text {auto }}(0.6 \%)$ performing best. For lung cases, WL and $\mathrm{RS}_{\text {auto }}$ methods generated dose distributions most similar to the ground truth.

Conclusion The $\mathrm{RS}_{\text {auto }}$ density override approach is an attractive option for CTN adjustments for a variety of anatomical sites. $\mathrm{RS}_{\text {auto }}$ methods were validated, resulting in dose calculations that were consistent with those calculated on diagnostic-quality CT images, for CBCT images acquired of the lung, for patients receiving pelvic RT in cases without excess adipose tissue, and for $\mathrm{H} \& \mathrm{~N}$ cases.

Keywords Cone beam computed tomography - Adaptive radiation therapy, ART $\cdot$ Dose calculation · Hounsfield units $\cdot$ Density

Vergleich unterschiedlicher CT-Kalibrierungsmethoden zur Dosisberechnung auf Basis der Kegelstrahlcomputertomographie

\section{Zusammenfassung}

Ziel Ziel dieser Arbeit ist der Vergleich und die Validierung mehrerer CT-Kalibrierungsmethoden zur Dosisberechnung auf der Grundlage von Kegelstrahlcomputertomographie(C BCT)-Aufnahmen. 
Methoden Bei 4 Becken-, 3 Lungen- und 4 Kopf-HalsPatienten wurde die Genauigkeit der Dosisberechnung auf der Basis von CBCT-Aufnahmen für die folgenden Ansätze untersucht: einerseits Rekonstruktion der CBCT-Aufnahmen mithilfe eines Streukorrekturalgorithmus $\left(\mathrm{CBCT}_{\mathrm{r}}\right)$ und andererseits 3 verschiedene Methoden zur expliziten $\mathrm{Zu}$ weisung der Elektronendichten auf Basis des CBCT-Datensatzes (W: Zuordnung von Wasserdichte auf dem gesamten CBCT, WB: Zuordnung von entweder Wasser- oder Knochendichte, WL: Zuordnung von entweder Wasser- oder Lungendichte) sowie der in einem kommerziellen Planungssystem implementierten Methode der automatischen Zuordnung von 6 Dichtestufen auf Basis der CBCT-Hounsfield-Werte $\left(\mathrm{RS}_{\text {auto }}\right)$. Als Grundlage zur Evaluierung der Methoden diente die Dosisverteilung, welche anhand einer am gleichen Tag wie die CBCT aufgenommenen Planungs-CT berechnet wurde. Die Genauigkeit der einzelnen Ansätze wurde anhand von Dosis-Volumen-Statistiken und lokalen Dosisunterschieden beurteilt.

Ergebnisse Bei den Datensätzen mit Tumoren im Beckenbereich ist die mittlere Dosisabweichung für alle Kalibrierungsmethoden unter $1,5 \%$, wobei $\mathrm{RS}_{\text {auto }}$ in einer überdurchschnittlichen Abweichung für Patienten mit einem höheren Anteil von Fettgewebe resultiert. Die mittlere Abweichung für Kopf-Hals-Patienten beträgt unter $1,0 \%$, wobei $\mathrm{CBCT}_{\mathrm{r}}$ $(0,5 \%)$ und $\mathrm{RS}_{\text {auto }}(0,6 \%)$ am besten abschneiden. WL und $\mathrm{RS}_{\text {auto }}$ resultieren für die Patienten mit Lungentumoren in Dosisverteilungen, welche der Referenzdosisverteilung entsprechen.

Schlussfolgerung $\mathrm{RS}_{\text {auto }}$ zur Kalibrierung von CBCT-Aufnahmen zur Dosisberechnung ist eine aussichtsreiche Methode für die untersuchten Indikationen. Es wurde gezeigt, dass mithilfe von $\mathrm{RS}_{\text {auto }}$ die Dosisberechnung auf der Basis von CBCT-Aufnahmen von Lungen- und Kopf-Hals-Erkrankungen sowie für Tumoren im Beckenbereich bei Patienten ohne ein Übermaß an Fettgewebe in einer akkuraten Dosisverteilung resultiert.

Schlüsselwörter Cone-Beam-CT · Adaptive Strahlentherapie $\cdot$ Dosisberechnung $\cdot$ HounsfieldEinheiten · Dichte

Since its introduction, cone beam CT (CBCT) has been mainly utilized for image-guided radiotherapy (IGRT) [14]. CBCT images generated prior to treatment can also be used for adaptive radiotherapy (ART) [5, 6] in various ways, ranging from using the $\mathrm{CBCT}$ to estimate the dosimetric effect of patient weight loss, CBCT assessment in order to choose a "plan-of-the-day," to dose recalculation and treatment plan generation on CBCT images [7-11]. Commercially available treatment planning systems (TPSs) are now able to implement ART strategies.
CBCT image quality is dependent on acquisition parameters and, compared with diagnostic-quality planning CT images, exhibit increased artifacts and poorer image contrast owing to increased scatter, which itself is dependent on the size of the scanned object/patient [12-14]. CBCT images (CBCTs) generated using XVI on Elekta treatment units are not in true $\mathrm{CT}$ numbers (CTNs) and, therefore, in their original form, such CBCTs cannot be used directly for accurate dose computation [12]. In order to be able to perform CBCT-based ART, it is important that dose can be calculated accurately (i.e., consistent with that calculated on diagnostic $\mathrm{CT}$ images) on the $\mathrm{CBCT}$ image; various methods have been suggested for CTN adjustment in order to achieve this [12, 15-21].

Strategies for CTN adjustment include calibration of the CBCT image voxel values with physical density following CBCT acquisitions of phantoms with inserts of differing densities [15-19]. Richter et al. [17] proposed a method based on average CBCT values for separate treatment sites in order to generate population-specific conversion curves for brain, head and neck $(\mathrm{H} \& \mathrm{~N})$, thorax, and prostate treatment sites. Apart from taking a large amount of time to define, conversion curves are subject to errors caused by image artifacts and patient variability, resulting in differences of $5 \%$ and larger between doses calculated on CBCTs compared with CT-based calculation [12, 17]. Patient-specific conversion curves have also been investigated [20]. However, these methods are still prone to dosimetric errors resulting from $\mathrm{CBCT}$ artifacts.

Poludniowski et al. have shown that differences, between the doses on CBCT and CT, of less than $2.5 \%$ can be achieved when the CBCT has been reconstructed after scatter correction of the individual projections [20, 22]. The implementation of such an approach can be slow and difficult to introduce into a clinical workflow. Commercially available systems that generate CBCTs in CTNs by using sophisticated scatter-correction algorithms are starting to become commercially available in recent software releases. However, the accuracy of dose calculation on such images has yet to be validated.

Relatively unsophisticated methods, such as density overrides of regions of interest, can be used to populate CBCTs with density values that result in acceptable dose calculation accuracy $[12,20,21]$. Such strategies can be fast to implement and can easily be adapted into clinical workflows. These techniques are not as sensitive to the problems, such as image artifacts, that the more sophisticated methods struggle to cope with.

Unlike other studies presented for density assignment methods [12, 21] and other approaches [15-20, 23], we use radiotherapy planning $\mathrm{CTs}\left(\mathrm{PCT}_{\mathrm{CBCT}}\right)$ acquired on the same day as the CBCT as the ground truth for dose calculation. Thus, we have selected patients with minimal anatomical 
differences between CBCTs and planning CTs, eliminating the need for, and uncertainties associated with, deformable image registration (DIR) [24-27]. Using the original planning $\mathrm{CT}\left(\mathrm{PCT}_{\text {orig }}\right)$, which may have been captured weeks before the CBCT, as the ground truth for dose calculation is unsuitable in the context of ART because establishing an estimate of the difference in dose between that calculated on the $\mathrm{PCT}_{\text {orig }}$ and $\mathrm{CBCT}$ is itself a subject of importance. We have investigated three anatomical sites: $H \& N$, pelvis (prostate and bladder), and lung.

The aim of this work was to assess the CBCT dose calculation accuracy for density override approaches and to compare this with the physics-based scatter correction of Poludniowski $[20,22]$. We evaluated the accuracy of the RayStation TPS (V3.99, RaySearch Laboratories, Stockholm, Sweden) density override approach and compared it with more simple density override methods investigated in the literature [12]. We aimed to establish, for different anatomical locations, the methods that were best in terms of CBCT dose calculation accuracy. The methods for CTN adjustment investigated in this work are transferable to dose calculation on magnetic resonance (MR) images and could, therefore, be of interest to MR-based ART [28].

\section{Patients and methods}

We selected patients who had a CBCT and diagnostic CT (both with the same immobilization and/or support devices) on the same day. The study consisted of four H\&N cases, four pelvis cases (two prostate and two bladder treatment sites), and three lung cases (two left upper lobe GTVs, one right upper lobe GTV). The RayStation TPS was used for dose calculations, using a collapsed cone algorithm. The median time difference between the $\mathrm{CBCT}$ and $\mathrm{PCT}_{\mathrm{CBCT}}$ was 59 min (range: 17-215 min).

CBCT dose calculation

The CBCTs were acquired with Elekta XVI-V4.5 (XVI Elekta, Stockholm, Sweden); the scanning parameters are displayed in Table 1. To calculate dose on the CBCT images, an external patient contour was defined. First, an external contour of the CBCT image was defined using the TPS automated algorithm that uses a thresholding technique based on the image data. Small holes were then removed from the threshold-generated volume and finally contours not associated with the largest object were deleted. Next, a field-of-view (FOV) region of interest (ROI) was defined for the CBCT image set (see Table 1 for FOV parameters). Finally, with a priori knowledge of the planning CT scan, an automated algorithm (available in the TPS) updated the external contour of the CBCT (where necessary) to extend beyond the CBCT FOV. A missing tissue ROI (with density $\left.=1 \mathrm{~g} / \mathrm{cm}^{3}\right)$ is created and is defined as the newly generated external ROI exterior to the FOV ROI. Figure 1 (top left) displays the FOV, external, and missing tissue ROIs for an $\mathrm{H} \& \mathrm{~N} C B C T$ image as a red contour, green contour, and gray color wash, respectively.

\section{CTN adjustment using CBCT reconstruction}

Following Poludniowski [20, 22, 29], CBCT reconstructions $\left(\mathrm{CBCT}_{\mathrm{r}}\right)$ were made after scatter correction. The software developed in-house explicitly simulates and corrects for scatter in the projection images using the patients $\mathrm{PCT}_{\text {orig }}$ as a prior [20,22]. A patient-independent look-up table was applied to the reconstructed images in order to convert the reconstructed CTNs into physical density [29].

\section{CTN adjustment using density overrides}

The "water only" (W) method, where all tissue was assigned as water $\left(1 \mathrm{~g} / \mathrm{cm}^{3}\right)$, was investigated in all three treatment sites. For pelvis and lung cases, "water-and-bone" (WB) and "water-and-lung" (WL) techniques were also investigated, respectively. For $\mathrm{WB}$, the $\mathrm{CBCT}$ voxels were assigned as either water or bone. Bone ROIs were generated for each CBCT image using a segmentation algorithm based on gray value thresholds on the $\mathrm{PCT}_{\text {orig }}$ data and copying the resulting ROIs onto the CBCT image. The bone ROIs were edited if necessary, to ensure they were appropriate with reference

Table 1 CBCT scanning parameters for the three anatomical sites investigated in this study

\begin{tabular}{lccccccc}
\hline Protocol name & $\mathrm{KeV}$ & $\mathrm{mA}$ & $\mathrm{mS}$ & FOV $(\mathrm{cm})$ & $\begin{array}{l}\text { Scan length } \\
(\mathrm{cm})\end{array}$ & Filter & $\begin{array}{l}\text { Nominal dose } \\
\text { CTDI (mGy) }\end{array}$ \\
\hline Chest S20 & 120 & 25 & 40 & 26 & 26 & F0 (none) & 9.00 \\
Head and neck & 100 & 10 & 10 & 26 & 26 & F0 (none) & 0.45 \\
S20 & & & 40 & 40 & 13 & F1 (bowtie) & 27.00 \\
Pelvis M10 & 120 & 100 & 40 & & \\
\hline
\end{tabular}

CTDI computed tomography dose index.

${ }^{a}$ The H\&N and lung patients were scanned with a field of view (FOV) of $26 \mathrm{~cm}$ allowing a partial gantry rotation for acquisition, but limiting the full patient contour (i.e., missing the shoulders on the H\&N patients and only seeing the treated sides of the lung patients), whereas acquisitions for the pelvis patients were made with the $40-\mathrm{cm}$ FOV. 
Fig. 1 The RayStation treatment planning system (TPS) cone beam computed tomography (CBCT) $\mathrm{CT}$ number $(\mathrm{CTN})$ adjustment method. Top row from left: Sagittal slice of a CBCT image of an $\mathrm{H} \& \mathrm{~N}$ cancer patient viewed within the TPS; the CBCT after density assignment by the TPS (regions assigned as bone are shown as yellow, for example); CTN-density table generated for the CBCT image. Bottom row, left: Typical CBCT acquisition of a patient with a tumor in their right lung and (right) the same CBCT image but with the field of view (red contour), external (green contour), and left lung (orange contour) regions of interest displayed
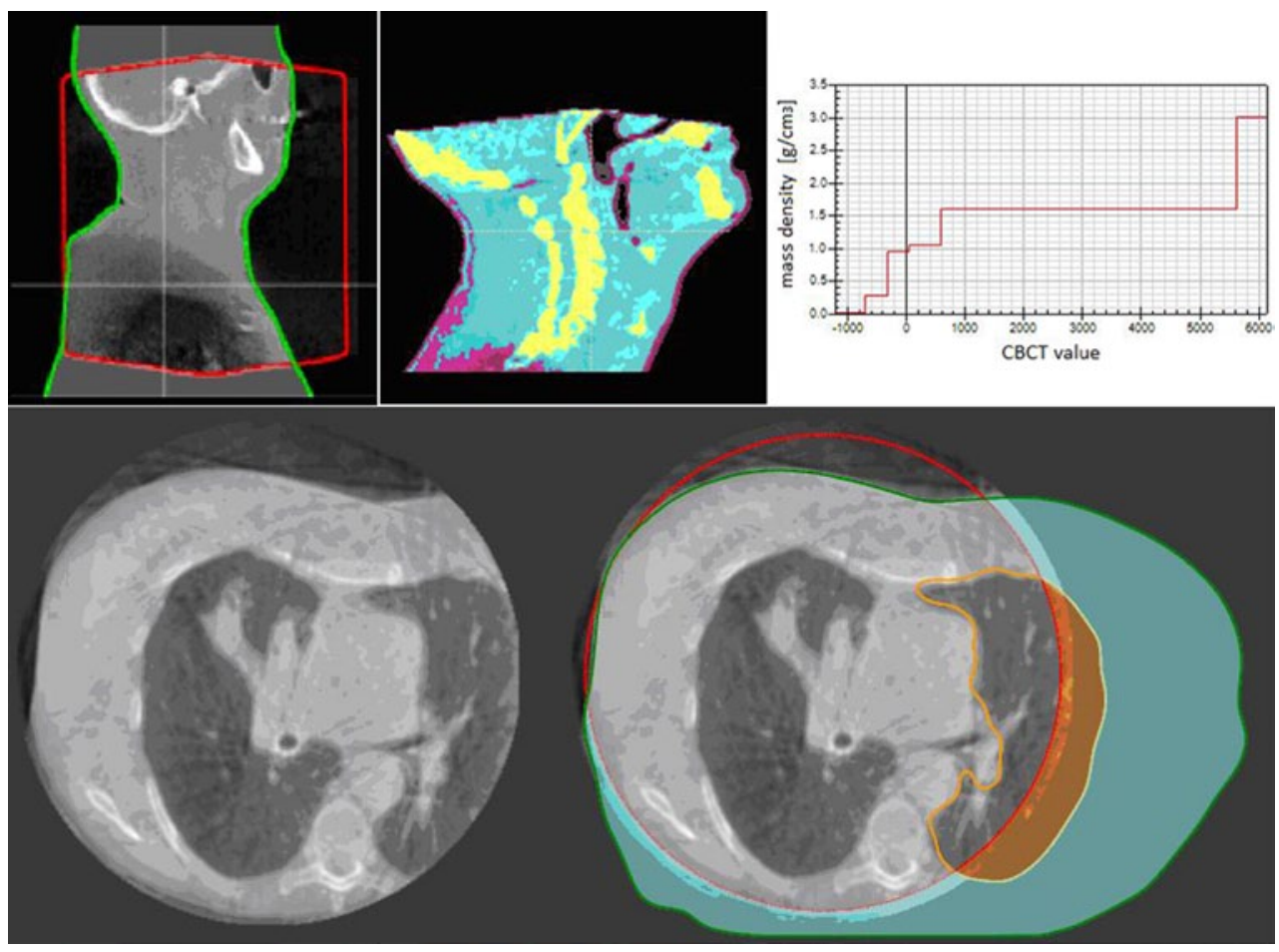

Table 2 The different CTN adjustment methods investigated in this study

\begin{tabular}{|c|c|c|}
\hline Method name & Method description & Sites used in study \\
\hline $\mathrm{CBCT}_{\mathrm{r}}$ & $\begin{array}{l}\text { CBCT reconstruction } \\
\text { after scatter correction } \\
\text { Poludniowski }\end{array}$ & Pelvis, H\&N, lung \\
\hline W & $\begin{array}{l}\text { All tissue was assumed to } \\
\text { be water }\left(1 \mathrm{~g} / \mathrm{cm}^{3}\right)\end{array}$ & Pelvis, H\&N, lung \\
\hline WB & $\begin{array}{l}\mathrm{CBCT} \text { voxels assigned as } \\
\text { either water or bone }\end{array}$ & Pelvis \\
\hline WL & $\begin{array}{l}\mathrm{CBCT} \text { voxels assigned as } \\
\text { either water or lung }\end{array}$ & Lung \\
\hline $\mathrm{RS}_{\text {auto }}$ & $\begin{array}{l}\text { CBCT voxels auto- } \\
\text { matically binned into six } \\
\text { density levels }\end{array}$ & Pelvis, H\&N, lung \\
\hline
\end{tabular}

$\overline{C B C T_{r} \text { physics-based scatter correction methods, } W \text { assignment of }}$ water density to the entire CBCT, $W B$ assignment of either water or bone density, $W L$ assignment of either water or lung density, $R S_{\text {auto }}$ RayStation TPS, $H \& N$ head and neck.

to the CBCT image. The $\mathrm{PCT}_{\text {orig }}$ bone ROIs were additionally used to establish an appropriate density to assign to the CBCT bone ROI. The average CTN of bone ROIs on PCT $\mathrm{P}_{\text {orig }}$ was 1,400 , which was similar to previously reported data [30] and equivalent to $1.35 \mathrm{~g} / \mathrm{cm}^{3}$. This density was used for all cases included in this study. In the WL technique, the patient was assumed to be made up of either water or lung tissue $\left(0.2 \mathrm{~g} / \mathrm{cm}^{3}\right)$, where the density of lung was calculated using similar methods as those used to determine the pelvic bone density.

More sophisticated density override techniques were investigated using the CBCT density-assignment tools avail- able in the TPS where six different set densities (air, lung, adipose tissue, connective tissue, cartilage/bone, and higher density for prosthesis) are assigned to the CBCT image. This is achieved by binning the CBCT image histogram into six density levels. The CBCT image thresholds are individual to each patient whereas the assigned density values are fixed (Fig. 1). An automatic algorithm $\left(\mathrm{RS}_{\text {auto }}\right)$ extracts the various density thresholds by approximating the CBCT image histogram with two normal distributions which were then interrogated to determine the threshold values. The physical densities applied by the TPS to the various tissue types were: air $=0.00121 \mathrm{~g} / \mathrm{cm}^{3}$, lung $=0.26 \mathrm{~g} / \mathrm{cm}^{3}$, adipose $=0.95 \mathrm{~g} / \mathrm{cm}^{3}$, connective tissue $=1.05 \mathrm{~g} / \mathrm{cm}^{3}$, cartilage $/$ bone $=1.6 \mathrm{~g} / \mathrm{cm}^{3}$, and other (such as prosthetic hip) $=3 \mathrm{~g} / \mathrm{cm}^{3}$. The consideration of the other override approaches (W, WL, WB) provides a useful benchmark and understanding for the more complex $\mathrm{RS}_{\text {auto }}$ methodology. Table 2 outlines the $\mathrm{CTN}$ adjustment techniques investigated in this study.

\section{Limited FOV for lung CBCTs}

At our institution, the FOV for CBCT acquisitions for lung patients does not encompass the entire patient contour (Table 1, Fig. 1). When performing CTN adjustment using $\mathrm{CBCT}_{\mathrm{r}}$ or $\mathrm{RS}_{\text {auto }}$, it may, therefore, be necessary to perform an additional density override to the contralateral lung. As well as applying a density override of $1 \mathrm{~g} / \mathrm{cm}^{3}$ to the missing tissue ROI (region shaded turquoise in Fig. 1), an additional density override of $0.2 \mathrm{~g} / \mathrm{cm}^{3}$ was applied to the left lung outside of the FOV (shaded orange in Fig. 1). If CBCT scans 
Fig. 2 Dose difference maps for between doses calculated on $\mathrm{CBCT}$ images and the ground truth (PCTCBCT). From top row to bottom: (1) a pelvic case with anterior-posterior distance $\left(\mathrm{D}_{\mathrm{AP}}\right)$ $=23 \mathrm{~cm}$; (2) a pelvic case with $\mathrm{D}_{\mathrm{AP}}=32 \mathrm{~cm} ;(3)$ an $\mathrm{H} \& \mathrm{~N}$ case; and (4) a lung case. All dose difference maps are presented as a percentage of the prescribed dose

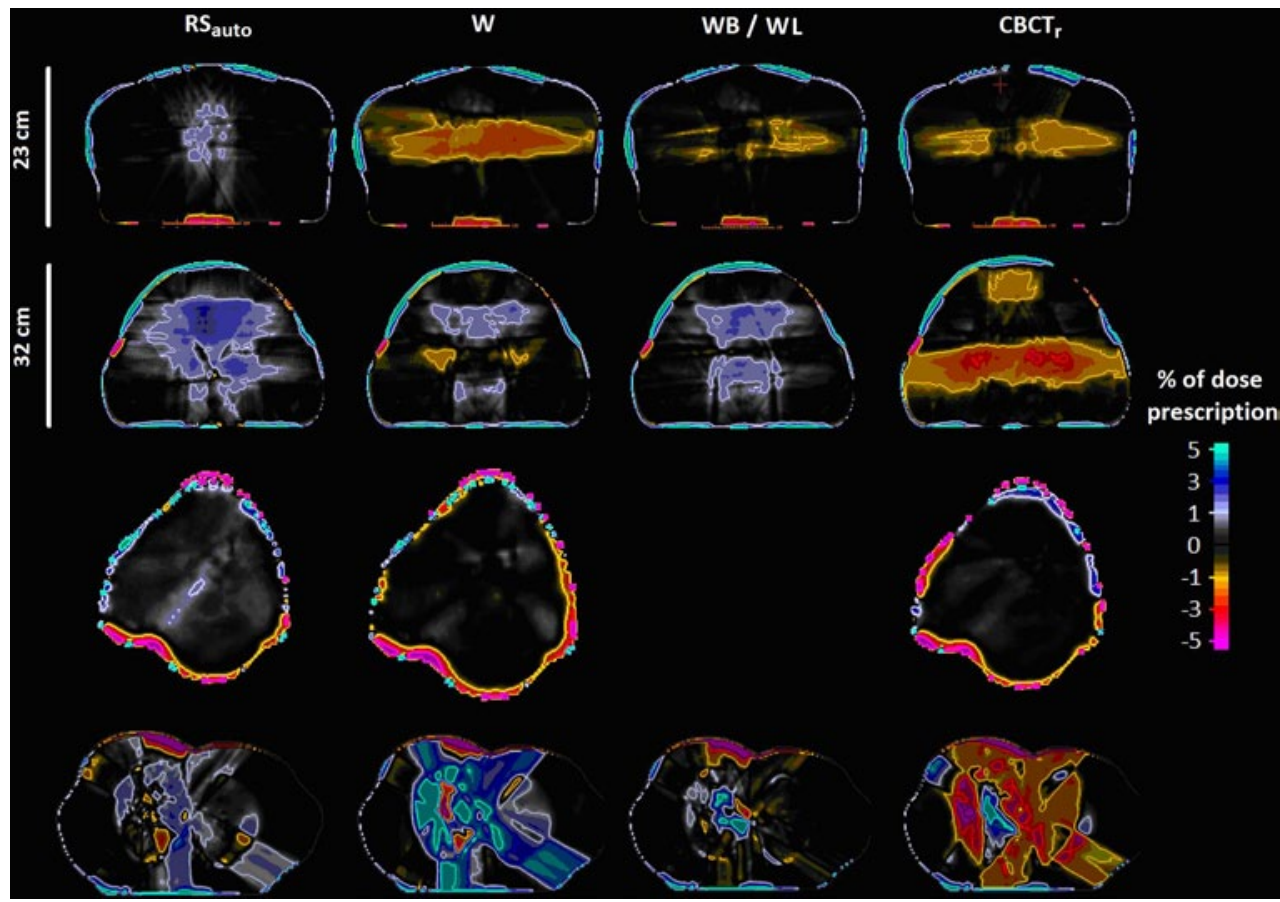

of the lung were to be routinely used for dose calculation, we would recommend using either a medium or large FOV to ensure the entire patient contour at the superior/inferior extent of the tumor was encompassed. This contralateral lung density issue has only a small effect on treatments where all beams enter ipsilaterally. However, for treatments where one (or more) beams entered contralaterally, the lung ROI from the planning CT scan was copied to the CBCT image and a density override was applied (Fig. 1).

\section{Data analysis}

Dose statistics and dose-difference maps were generated using the TPS. Dose-volume histogram (DVH) analysis was performed on ROIs that were propagated to the $\mathrm{CBCT}$ from the $\mathrm{PCT}_{\mathrm{CBCT}}$ scan.

\section{Results}

Differences in dose-volume statistics between that calculated on the $\mathrm{CBCT}$ and the ground truth, for all three treatment sites, are shown in Table 3 and Fig. 2.

Internal anatomy variability of a prostate case is illustrated in Fig. 3, demonstrating that the use of $\mathrm{PCT}_{\mathrm{CBCT}}$ as the ground truth is, in some instances, essential for meaningful DVH dosimetric comparison for CBCT dose calculation accuracy. Previous studies [12, 15-21, 23] of CBCT dose calculation accuracy have either used rigid (which may have been acquired weeks prior to the $\mathrm{CBCT}$ and therefore exhibit substantial anatomical differences) or deform- able image registrations (and their associated uncertainty) between the original planning CT and CBCT.

\section{Pelvis cases}

$\mathrm{RS}_{\text {auto }}$ tended to underestimate the proportion of lower-density tissue present in larger patients (Fig. 4), corresponding to an underestimation of the dose. This underestimation has been observed in a larger cohort of pelvic cases that were not used in this dose validation study as they did not have a CBCT and CT acquired on the same day. However, for patients with anterior-posterior distance $\left(\mathrm{D}_{\mathrm{AP}}\right)<25 \mathrm{~cm}$, $\mathrm{RS}_{\text {auto }}$ resulted in the most accurate dose distributions of all CTN adjustment methods with an average of $0.7 \%$ absolute difference in dose when compared with the ground truth. Furthermore, for patients with $\mathrm{D}_{\mathrm{AP}}<25 \mathrm{~cm}$, the WB method worked well with the assignment of water $\left(1 \mathrm{~g} / \mathrm{cm}^{3}\right)$ to all non-bone tissue, being adequate in such cases as these patients commonly have similar amounts of lower-density $\left(0.95 \mathrm{~g} / \mathrm{cm}^{3}\right)$ and connective $\left(1.05 \mathrm{~g} / \mathrm{cm}^{3}\right)$ tissue. However, for patients with higher proportions of adipose tissue, the WB method did not perform as well. The W method performed better than WB for patients with $\mathrm{D}_{\mathrm{AP}}>25 \mathrm{~cm}$, with the additional adipose tissue being balanced out by the higher-density bone. The $\mathrm{CBCT}_{\mathrm{r}}$ method was unable to accurately reconstruct higher-density material for the larger patient, resulting in a similar effect to that observed with the $\mathrm{W}$ method for patients with $\mathrm{D}_{\mathrm{AP}}<25 \mathrm{~cm}$. For this treatment site, the $\mathrm{RS}_{\text {auto }}$ method produced the best results when compared with the ground truth, with an average absolute difference of $0.7 \%$ (range: $0.1-2.5 \%$ ) when only consider- 
Table 3 Dose difference statistics of target and organs at risk as calculated on the CBCT compared with the ground truth

\begin{tabular}{|c|c|c|c|c|c|c|}
\hline & & $\begin{array}{l}\mathrm{CBCTr} \\
\text { mean\%diff } \\
(\% \text { range })\end{array}$ & $\begin{array}{l}\text { W } \\
\text { mean\%diff } \\
\text { (\% range) }\end{array}$ & $\begin{array}{l}\text { WB } \\
\text { mean\%diff } \\
\text { (\% range) }\end{array}$ & $\begin{array}{l}\text { WL } \\
\text { mean\%diff } \\
(\% \text { range })\end{array}$ & $\begin{array}{l}\mathrm{RS}_{\text {auto }} \\
\text { mean\%diff } \\
\text { (\% range) }\end{array}$ \\
\hline \multicolumn{7}{|l|}{ Pelvic treatments } \\
\hline \multirow[t]{4}{*}{ CTV } & $\mathrm{D}_{\text {median }}$ & $0.8(0.5-1.2)$ & $0.3(-1.3-1.7)$ & $-1.0(-1.6--0.1)$ & & $-1.3(-2.6--0.1)$ \\
\hline & $\mathrm{D}_{95 \%}$ & $0.8(0.0-2.0)$ & $0.0(-2.4-1.4)$ & $-1.5(-2.7--0.4)$ & & $-1.5(-3.7--0.2)$ \\
\hline & $\mathrm{D}_{98 \%}$ & $0.8(0.0-2.0)$ & $-0.1(-2.6-1.4)$ & $-1.5(-2.8--0.4)$ & & $-1.7(-3.9--0.4)$ \\
\hline & $\mathrm{D}_{2 \%}$ & $1.2(0.7-1.5)$ & $0.7(-0.2-1.9)$ & $-0.7(-1.4--0.2)$ & & $-0.8(-1.2-0.1)$ \\
\hline Femoral heads & $\mathrm{D}_{50 \%}$ & $2.4(1.6-3.1)$ & $0.9(-1.2-3.9)$ & $-1.1(-1.8--0.5)$ & & $-0.7(-1.5--0.1)$ \\
\hline Rectum & $\mathrm{D}_{50 \%}$ & $1.4(-0.2-4.2)$ & $0.2(-1.1-1.1)$ & $-1.0(-1.8-0.5)$ & & $0.7(-1.3-0.6)$ \\
\hline Bladder & $\mathrm{D}_{\text {mean }}$ & $0.4(0.0-0.8)$ & $0.1(-0.3-0.4)$ & $-1.0(-1.2--0.8)$ & & $-1.5(-1.6--1.4)$ \\
\hline Average $^{a}$ & & $1.4(-0.3-4.2)$ & $0.0(-2.6-3.9)$ & $-1.0(-2.8-0.5)$ & & $-1.1(-3.9-2.0)$ \\
\hline Absolute average $^{a}$ & & $1.4(0.0-4.2)$ & $0.7(0.0-3.9)$ & $1.0(0.1-2.8)$ & & $1.2(0.0-3.9)$ \\
\hline \multicolumn{7}{|c|}{ Head and neck treatments } \\
\hline \multirow[t]{4}{*}{ CTV } & $\mathrm{D}_{\text {median }}$ & $0.3(-0.1-1.0)$ & $0.4(0.0-1.3)$ & & & $-0.1(-0.6-0.1)$ \\
\hline & $\mathrm{D}_{95 \%}$ & $0.5(0.1-0.9)$ & $1.9(0.2-4.7)$ & & & $0.2(-0.3-1.0)$ \\
\hline & $\mathrm{D}_{98 \%}$ & $0.4(0.0-0.9)$ & $0.4(0.0-0.8)$ & & & $-0.1(-0.3-0.0)$ \\
\hline & $\mathrm{D}_{2 \%}$ & $0.3(-0.2-1.0)$ & $0.8(0.1-1.4)$ & & & $-0.1(-0.4-0.2)$ \\
\hline Spinal cord & $\mathrm{D}_{2 \%}$ & $0.7(0.0-1.7)$ & $1.4(1.0-2.5)$ & & & $0.5(-0.1-1.0)$ \\
\hline Brain stem & $\mathrm{D}_{2 \%}$ & $0.5(-0.6-1.4)$ & $0.5(-1.7-1.6)$ & & & $-0.5(-2.5-0.6)$ \\
\hline Parotids & $\mathrm{D}_{\text {mean }}$ & $0.5(0.0-1.1)$ & $0.4(-0.5-1.4)$ & & & $-0.3(1.5-0.4)$ \\
\hline Average $^{b}$ & & $0.4(-0.6-1.7)$ & $0.8(-1.7-4.7)$ & & & $-0.1(-2.5-1.0)$ \\
\hline Absolute average $^{\mathrm{b}}$ & & $0.5(0.0-1.7)$ & $1.0(0.0-4.7)$ & & & $0.6(0.1-2.5)$ \\
\hline \multicolumn{7}{|l|}{ Lung treatments } \\
\hline \multirow[t]{4}{*}{ CTV } & $\mathrm{D}_{\text {median }}$ & $2.4(1.9-2.9)$ & $-6.8(-7.7--6.1)$ & & $\begin{array}{l}0.4 \\
(-0.6-0.9)\end{array}$ & $-1.3(-2.1--0.8)$ \\
\hline & $\mathrm{D}_{95 \%}$ & $0.0(-1.7-1.7)$ & $-7.0(-7.4--6.5)$ & & $\begin{array}{l}0.5 \\
(-2.0-1.8)\end{array}$ & $-1.1(-1.7--0.7)$ \\
\hline & $\mathrm{D}_{98 \%}$ & $-1.3(-4.8-2.2)$ & $-6.9(-7.1--6.6)$ & & $\begin{array}{l}0.3 \\
(-2.7-2.2)\end{array}$ & $-1.0(-1.5--0.7)$ \\
\hline & $\mathrm{D}_{2 \%}$ & $3.0(1.9-4.1)$ & $-6.2(-6.6--5.9)$ & & $\begin{array}{l}0.8 \\
(-0.1-1.4)\end{array}$ & $-0.9(-2.3-0.5)$ \\
\hline Heart & $\mathrm{D}_{\text {mean }}$ & $-0.2(-3.4-2.9)$ & $-3.4(-8.7-1.7)$ & & $\begin{array}{l}-0.9 \\
(-3.3-1.2)\end{array}$ & $-2.2(-4.0--0.8)$ \\
\hline Lungs & $\mathrm{D}_{\text {mean }}$ & $2.9(-1.0-8.0)$ & $-7.8(-12.7-4.6)$ & & $\begin{array}{l}0.4 \\
(-1.6-1.6)\end{array}$ & $-1.8(-3.9--0.3)$ \\
\hline Spinal cord & $\mathrm{D}_{2 \%}$ & $3.7(3.0-4.4)$ & $-2.4(-5.7-1.4)$ & & $1.1(0.0-1.7)$ & $-0.8(-1.9-0.5)$ \\
\hline Average $^{c}$ & & $1.5(-4.8-8.0)$ & $-5.9(-12.7-1.7)$ & & $\begin{array}{l}0.2 \\
(-3.3-1.8)\end{array}$ & $-1.4(-4.0-0.5)$ \\
\hline Absolute average $^{c}$ & & $2.8(1.0-8.0)$ & $6.1(1.4-12.7)$ & & $1.3(0.0-3.3)$ & $1.4(0.3-4.0)$ \\
\hline
\end{tabular}

$W$ water, $W B$ water or bone, $W L$ water or lung, $C T V$ clinical target volume.

${ }^{a}$ Average values are the mean of the dose difference statistics for all CTVs $\left(\mathrm{D}_{98 \%}, \mathrm{D}_{95 \%}\right.$, mean, $\left.\mathrm{D}_{50 \%}, \mathrm{D}_{2 \%}\right), \mathrm{D}_{50 \%}$ for femoral heads, $\mathrm{D}_{\text {mean }}$ for bladder, $\mathrm{D}_{50 \%}$ and $\mathrm{D}_{2 \%}$ for rectum.

${ }^{b}$ The average values are the mean of the dose difference statistics for all CTVs $\left(\mathrm{D}_{95 \%}\right.$, mean, $\left.\mathrm{D}_{50 \%}, \mathrm{D}_{2 \%}\right), \mathrm{D}_{50 \%}$ for parotids, $\mathrm{D}_{2 \%}$ for brain stem and spinal cord.

'The average values are the mean of the dose difference statistics for the CTV $\left(\mathrm{D}_{98 \%}, \mathrm{D}_{95 \%}\right.$, mean, $\left.\mathrm{D}_{50 \%}, \mathrm{D}_{2 \%}\right)$, mean dose to heart and healthy lung, $\mathrm{D}_{2 \%}$ for spinal cord.

ing patients with $\mathrm{D}_{\mathrm{AP}}<25 \mathrm{~cm}$. For CBCT images acquired around the pelvis of patients with $\mathrm{D}_{\mathrm{AP}}<25 \mathrm{~cm}$, we therefore recommend using the $\mathrm{RS}_{\text {auto }}$ method for CTN adjustment. However, the WB and W methods worked with sufficient accuracy [20] for patients with $\mathrm{D}_{\mathrm{AP}}<25 \mathrm{~cm}$ and $>25 \mathrm{~cm}$, respectively.

\section{$\mathrm{H} \& \mathrm{~N}$ cases}

All three methods investigated for CTN adjustment for $\mathrm{H} \& \mathrm{~N}$ cases resulted in acceptable [20] average absolute dose deviations of $\leq 1.0 \%$ when compared with the ground truth, with $\mathrm{CBCT}_{\mathrm{r}}(0.5 \%)$ and $\mathrm{RS}_{\text {auto }}(0.6 \%)$ performing best. For CBCT images acquired of the $\mathrm{H} \& \mathrm{~N}$, on the basis of the results presented in Table 3 , its integration within a 

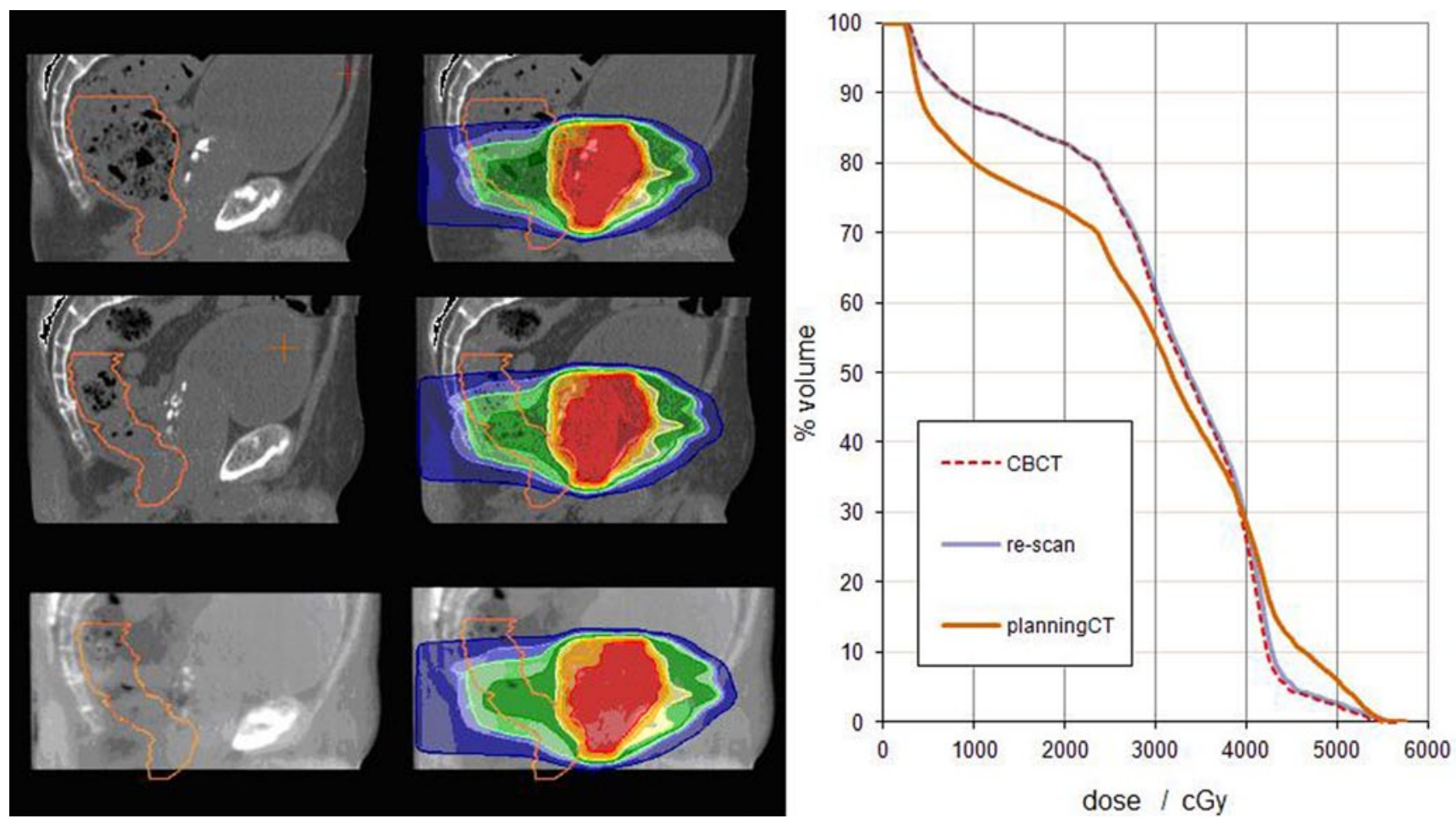

Fig. 3 Left: Sagittal images of a prostate RT treatment plan with dose calculated on (top) the $\mathrm{PCT}_{\text {orig }}$ (middle) $\mathrm{PCT}_{\mathrm{CBCT}}$; and (bottom) the CBCT. The rectum is shown as an orange contour and the dose is shown in color wash relative to the prescribed dose. Right: dose-volume histogram of the rectum for the dose calculated on the $\mathrm{PCT}_{\text {or }}$ scan (orange line); the $\mathrm{PCT}_{\mathrm{CBCT}}$ (blue line); and the $\mathrm{CBCT}$ (red dashed

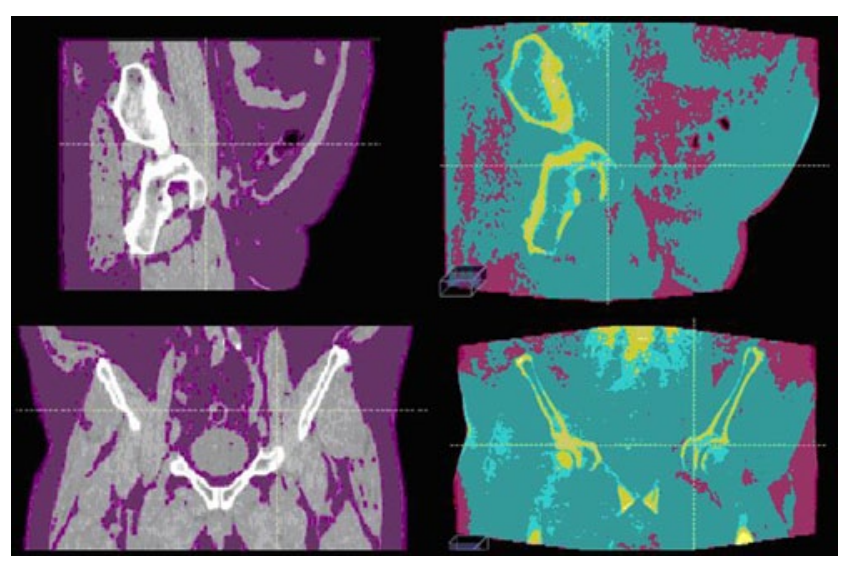

Fig. 4 Sagittal (top row) and coronal slices (bottom row) of a pelvic case with a high proportion of adipose tissue. From left to right: the $\mathrm{PCT}_{\mathrm{CBCT}}$ with tissue density $<0.95 \mathrm{~g} / \mathrm{cm}^{3}$ colored purple, and CBCT with $\mathrm{RS}_{\text {auto }} \mathrm{CTN}$ adjustment. In the CBCT images purple, turquoise, and yellow represent adipose $\left(0.95 \mathrm{~g} / \mathrm{cm}^{3}\right)$, connective tissue $(1.05 \mathrm{~g} /$ $\left.\mathrm{cm}^{3}\right)$, and bone $\left(1.6 \mathrm{~g} / \mathrm{cm}^{3}\right)$, respectively. $P C T$ planning computed tomography, $C B C T$ cone beam CT, CTN CT number

TPS, and its ease and speed of implementation, we recommend using the $\mathrm{RS}_{\text {auto }}$ method for CTN adjustment. Larger dose differences were observed in the inferior part of the target at the level of the shoulders. Similar to the methods line). The RS method was used for CTN adjustment of the CBCT image. The rectum OAR was similar in size and shape on the CBCT and the $\mathrm{PCT}_{\mathrm{CBCT}}$ but was very different to that seen on the $\mathrm{PCT}_{\text {orig }}$ scan. $P C T$ planning computed tomography, $C B C T$ cone beam CT, $C T N$ CT number, $O A R$ organ at risk

employed for the contralateral lung ROIs (Fig. 1), this dose disagreement may be improved by generating additional bone ROIs for the shoulders to enable more accurate dose calculation on the CBCT data set.

\section{Lung cases}

Assuming that density overrides external to the FOV ROI were applied as per Fig. 1, the WL and $\mathrm{RS}_{\text {auto }}$ methods generated dose distributions most similar to $\mathrm{PCT}_{\mathrm{CBCT}}$ calculations, with average absolute dose differences of 1.3 and $1.4 \%$, respectively (Table 3 ). When the $\mathrm{RS}_{\text {auto }}$ or $\mathrm{CBCT}_{\mathrm{r}}$ methods were applied to VMAT treatments without applying additional contralateral lung density overrides, the average absolute dose difference increased to over $3.0 \%$. The $\mathrm{W}$ method performed poorly for lung cases as, unlike the other methods investigated for this site, it did not account for the lower-density lung tissue present. On the basis of the results presented in Table 3, for CBCT images acquired of the lung, we recommend using either the $\mathrm{RS}_{\text {auto }}$ or WL method for CTN adjustment. This study did not investigate the dependence of CBCT-based dose calculations on lung intra-fraction motion. 


\section{Discussion}

For the sites investigated in this study, the differences between the doses calculated on the CBCT compared with the diagnostic CT, when using $\mathrm{RS}_{\text {auto }}$, were similar or better than other reported results. Fotina et al. demonstrated that density override techniques applied to CBCT scans of the prostate resulted in dosimetric differences below $2 \%$, whereas the use of conversion curves to calculate dose on CBCT images has been shown to result in differences of up to $5 \%$ [12]. However, Richter et al. demonstrated that conversion curves specific to anatomical sites can result in comparable dose calculation accuracy with differences below $2 \%$ [17].

Owing to weight loss during radiotherapy for $\mathrm{H} \& \mathrm{~N}$ cancer $[7,24,31,32]$, for the $H \& N$ cases it was necessary to use the $\mathrm{PCT}_{\mathrm{CBCT}}$ as the input to the algorithm within the TPS used to define the CBCT body contour. When the $\mathrm{PCT}_{\text {orig }}$ was used, the large differences in the body contour between the $\mathrm{PCT}_{\mathrm{CBCT}}$ and $\mathrm{CBCT}$ meant dose differences between that calculated on the $\mathrm{CBCT}$ and the ground truth could not be assessed with confidence. The use of the $\mathrm{PCT}_{\mathrm{CBCT}}$ data as a priori knowledge for this validation study reduced the uncertainty of the shape of the CBCT body contour, resulting in more accurate estimations of CBCT dose. If CBCT scans of the $\mathrm{H} \& \mathrm{~N}$ were to be routinely used for dose calculation, we would recommend using either a medium or large FOV to ensure the entire patient contour at the superior/ inferior extent of the tumor was imaged in order to generate the entire patient contour on the CBCT image. Cupping artifacts can have a large effect on CBCT dose calculations, and the degree to which they affect the dose depends on the CTN adjustment method used. CBCT-density look-up table techniques are the most sensitive in this context. However, CBCT dose distributions generated using density override methods, such as those discussed in this study, are also affected by image quality with increasing sensitivity with increasing number of density bins. However, the results indicate that binning the CBCT voxels into six densities is an appropriate methodology that results in dose distributions that are sufficiently accurate. As well as automatically defining the density threshold levels, the TPS allows them to be manually defined by the user. Especially important for larger-sized patients, this approach could be used to ensure an appropriate proportion is assigned as lower-density adipose tissue. However, tissue assignment as a manual process is subject to error, which was not investigated in this study but will be explored in future work. Although this study involved a small number of cases in each of the three treatment sites, we believe that we have identified patients with minimal anatomic differences between the CBCT and $\mathrm{PCT}_{\mathrm{CBC}}$, enabling the accuracy of CBCT dose calculation to be assessed and for the $\mathrm{RS}_{\text {auto }}$ method to be validated.

\section{Conclusion}

We investigated various methods, including $\mathrm{RS}_{\text {auto }}$ that has not been assessed before, for CTN adjustment for CBCT dose calculations. $\mathrm{RS}_{\text {auto }}$ methods were validated, resulting in CBCT dose calculations for lung patients, for patients treated in the pelvis with $\mathrm{D}_{\mathrm{AP}}<25 \mathrm{~cm}$, and for $\mathrm{H} \& \mathrm{~N}$ cases, which were similar to those calculated on diagnostic CT images. Its implementation into a commercially available TPS makes the $\mathrm{RS}_{\text {auto }}$ approach the fastest and easiest-to-use of all methods investigated in this study and it can easily be performed as part of a typical clinical workflow. The differences reported in this study between the doses calculated on the CBCT compared with the ground truth, when using density overrides, were similar to other reported results [12] and better than those that use CBCT-density look-up table methods [12]. However, in this study we used a planning CT acquired on the same day as the CBCT as the ground truth in order to minimize the uncertainties often present in studies of this kind. Although the RayStation TPS was used for this work, the methods described could be replicated in other treatment planning systems. Furthermore, the density override methods for CTN adjustment that were investigated in this work are applicable and transferable to dose calculation on segmented MR images.

Acknowledgments This work was undertaken in The Royal Marsden NHS Foundation Trust, which received a proportion of its funding from the NHS Executive; the views expressed in this publication are those of the authors and not necessarily those of the NHS Executive. This work was supported by Cancer Research UK Programme Grants C7224/A13407 and C33589/A19727. The authors also acknowledge the support of the National Institute for Health Research Royal Marsden and Institute of Cancer Research Biomedical Research Centre.

Open Access This article is distributed under the terms of the Creative Commons Attribution License which permits any use, distribution, and reproduction in any medium, provided the original author(s) and the source are credited.

\section{Compliance with ethical guidelines}

Conflict of interest A. Dunlop reports grants from NIHR Biomedical Research Centre and grants from Cancer Research UK C7224/ A13407. D. McQuaid reports grants from NIHR Biomedical Research Centre and grants from Cancer Research UK C7224/A13407. J. Murray states that there are no conflicts of interest. K. Newbold has received honoraria for advisory roles for Genzyme, Astra Zeneca, and Eisai. S. Nill reports grants from NIHR Biomedical Research Centre, grants from Cancer Research UK Programme Grant C33589/A19727, during the conduct of the study. V.N. Hansen states that there are no conflicts of interest. C. Nutting reports grants from Cancer Research UK C7224/A13407. G. Poludniowski states that there are no conflicts of interest. K. Harrington reports grants from NIHR Biomedical Research Centre, grants from Cancer Research UK C7224/A13407, during the study, and outside the submitted work, grants from Merck, 
from Cellgene, from Oncolytics Biotech, and from Genelux Corporation. S. Bhide states that there are no conflicts of interest. U. Oelfke reports grants from NIHR Biomedical Research Centre, grants from Cancer Research UK Programme Grant C33589/A19727, and grants from EPSRC Platform Grant EP/H046526/1, during the study.

All patients in this study had previously taken part in separate protocols which were approved by the institutional review board and research ethics committee, for which informed consent had been obtained for use of data for research purposes.

\section{References}

1. Smitsmans MH, de Bois J, Sonke JJ et al (2005) Automatic prostate localization on cone-beam CT scans for high precision imageguided radiotherapy. Int J Radiat Oncol Biol Phys 63:975-984

2. Purdie TG, Bissonnette JP, Franks K et al (2007) Cone-beam computed tomography for on-line image guidance of lung stereotactic radiotherapy: localization, verification, and intrafraction tumor position. Int J Radiat Oncol Biol Phys 68:243-252

3. Moseley DJ, White EA, Wiltshire KL et al (2007) Comparison of localization performance with implanted fiducial markers and cone-beam computed tomography for on-line image-guided radiotherapy of the prostate. Int J Radiat Oncol Biol Phys 67:942-953

4. Jaffray D, Siewerdsen J, Wong J et al (2001) Flat-panel cone-beam computed tomography for image-guided radiation therapy. Int $\mathrm{J}$ Radiat Oncol Biol Phys 53:1337-1349

5. Vestergaars A, Muren L, Sondergaard J et al (2013) Adaptive plan selection vs. Re-optimisation in radiotherapy for bladder cancer: a dose accumulation comparison. Radiother Oncol 109:457-462

6. Pos F, Hulshof M, Lebesque J et al (2006) Adaptive radiotherapy for invasive bladder cancer: a feasibility study. Int J Radiat Oncol Biol Phys 64:862-868

7. Hansen E, Bucci M, Quivey J et al (2006) Repeat CT imaging and replanning during the course of IMRT for head-and-neck cancer. Int J Radiat Oncol Biol Phys 64:355-362

8. Schwartz D, Dong L (2011) Adaptive radiation therapy for head and neck cancer - can an old goal evolve into a new standard? J Oncol 2011. doi:10.1155/2011/690595. (Article ID 690595, 13 pages)

9. Chai X, van Herk M, Betgen A et al (2012) Automatic bladder segmentation on CBCT for multiple plan ART of bladder cancer using a patient-specific bladder model. Phys Med Biol 57:3945-3962

10. Létourneau D, Wong R, Moseley D et al (2007) Online planning and delivery technique for radiotherapy of spinal metastases using cone-beam CT: image quality and system performance. Int J Radiat Oncol Biol Phys 67:1229-1237

11. Létourneau D, Kaus M, Wong R et al (2008) Semiautomatic vertebrae visualization, detection, and identification for online palliative radiotherapy of bone metastases of the spine. Med Phys $35: 367-376$

12. Fotina I, Hopfgartner J, Stock M et al (2012) Feasibility of CBCTbased dose calculation: comparative analysis of HU adjustment techniques. Radiother Oncol 104:249-256

13. Kim S, Yoo S, Yin FF et al (2010) Kilovoltage cone-beam CT: comparative dose and image quality evaluations in partial and fullangle scan protocols. Med Phys 37:3648-3459

14. Stock M, Pasler M, Birkfellner W et al (2009) Image quality and stability of image- guided radiotherapy (IGRT) devices: a comparative study. Radiother Oncol 93:1-7

15. Yang Y, Schrwibmann E, Wang C et al (2007) Evaluation of onboard $\mathrm{kV}$ cone beam CT (CBCT)-based dose calculation. Phys Med Biol 52:685-705
16. Yoo S, Yin F-F (2006) Dosimetric feasibility of cone-beam CTbased treatment planning compared to CT-based treatment planning. Int J Radiat Oncol Biol Phys 66:1553-1561

17. Richter A, Hu Q, Steglich D et al (2008) Investigation of the usability of conebeam CT data sets for dose calculation. Radiat Oncol 3:42-55

18. Hatton J, McCurdy B, Greer P (2009) Cone beam computerized tomography: the effect of calibration of the Hounsfield unit to electron density on dose calculation accuracy for adaptive radiotherapy. Phys Med Biol 54:329-346

19. Hatton J, Greer P, Tang C et al (2011) Does the planning dosevolume histogram represent treatment doses in image-guided prostate radiation therapy? Assessment with cone-beam computerised tomography scans. Radiother Oncol 98:162-168

20. Poludniowski G, Evans P, Webb S (2012) Cone beam computed tomography number errors and consequences for radiotherapy planning: an investigation of correction methods. Int J Radiat Oncol Biol Phys 84:109-114

21. Onozato Y, Kadoya N, Fujita Y et al (2014) Evaluation of on-board cone beam computed tomography-based dose calculation with deformable image registration using Hounsfield unit modifications. Int J Radiat Oncol Biol Phys 89:416-423

22. Poludniowski G, Evans P, Hansen VN et al (2009) An efficient Monte-Carlo-based algorithm for scatter correction in $\mathrm{keV}$ conebeam CT. Phys Med Biol 54:3847-3864

23. Wang J, Hu W, Cai G et al (2013) Using corrected cone-beam CT image for accelerated partial breast irradiation treatment dose verification: the preliminary experience. Radiat Oncol 8:214-222

24.. Veiga C, McClelland J, Moinuddin S et al (2014) Toward adaptive radiotherapy for head and neck patients: feasibility study on CTto-CBCT deformable registration for "dose of the day" calculations. Med Phys 41:031703

25. Fortunati V, Verhaart R, Angeloni F et al (2014) Feasibility of multimodal deformable registration for head and neck tumor treatment planning. Int J Radiat Oncol Biol Phys 90:85-93

26. Brock K (2010) Results of a multi-institution deformable registration accuracy study (MIDRAS). Int J Radiat Oncol Biol Phys 76:583-596

27. Mencarelli A, van Kranen S, Hamming-Vrieze O et al (2014) Deformable image registration for adaptive radiation therapy of head and neck cancer: accuracy and precision in the presence of tumor changes. Int J Radiat Oncol Biol Phys 90:680-687

28. Lagendijk L, Raaymakers B, Raaijmakers AJ et al (2008) MRI/ linac integration. Radiother Oncol 86:25-29

29. Poludniowski G, Evans P, Kavanagh A et al (2011) Removal and effects of scatter-glare in cone-beam CT with an amorphous-silicon flat-panel detector. Phys Med Biol 56:1837-1851

30. Lee Y, Bollet M, Charles-Edwards G et al (2003) Radiotherapy treatment planning of prostate cancer using magnetic resonance imaging alone. Radiother Oncol 66:203-216

31. Barker J, Garden A, Ang KK et al (2004) Quantification of volumetric and geometric changes occuring during fractionated radiotherapy for head and neck cancer using an integrated CT/linear accelerator system. Int J Radiat Oncol Biol Phys 59:960-970

32. Marzi S, Pinnaro P, D’Alessio D et al (2012) Anatomical and dose changes of gross tumour volume and parotid glands for head and neck cancer patients during intensity-modulated radiotherapy: effect on the probability of xerostomia incidence. Clin Oncol (R Coll Radiol) 24:e54-e62 